\title{
506 - Methodologies used to study the feasibility, usability and effectiveness of social robots in clinical and social care settings for elderly adults \\ Aysan Mahmoudi, Mauricio Molinari, Henriette Van Der Roest, Manuel Franco
}

Background: In a search for innovative dementia care strategies, a new research fields have been dedicated to design social robots for older adults with cognitive impairments and dementia in order to promote their quality of life through communication and social interactions. Although the results are promising, the quality of studies on effectiveness of social robots in elderly adults is still low due to several methodological limitations (1). Then, we aim to review the methodologies used so far for conducting studies with social robots in clinical and social settings with people with dementia and elderly adults.

Method: Searches in Medline (PubMed), Web of Science, Psyclnfo, and Cinahl yielded 33 studies included in the final review after applying inclusion and exclusion criteria. Reviews were conducted by two authors independently.

Result: Across 33 papers, 23 different social robots were examined on elderly adults and PwD, investigating feasibility, usability and effectiveness. A variety of study designs such as RCTs, mixed methods, cross-sectional and cohort were employed. Individual and group-based interventions took place in private households, LTCFs and labs within a single session or multiple sessions. Data were collected through observation, questionnaires, interview and physiological tests. Most revealed improvements were found in mood, engagement and participation. However, improvements in QoL, depression and cognition were not significant.

Conclusion: Despite the promising results, due to the insufficient methodology, failures and ambitious findings remain within the many aspects of the enquiry. Appropriate RCT designs with large sample sizes and individual intervention sessions might be established for effectiveness studies. For feasibility/usability studies, a cohort design with long-term intervention would serve best. We also

recommend a mixed method of data collection for any of three effectiveness, feasibility and usability study aims. Multiple interaction sessions running for more than one month might help researches to draw significant results and reveal the long-term impact of the robots.

\section{7 - Stressful past events and emotional intelligence as predictors of Successful aging}

Cristina Buedo-Guirado, PHD, Cristina G. Dumitrache, Laura Rubio, PhD

\begin{abstract}
Objectives. It has been widely demonstrated that recent stressful events could impact wellbeing, nevertheless it is not clear whether stressful situations experienced in the pastand the internal resources used to deal with them can also influence older adult's well- being. The purpose of this study was to evaluate the influence of stressful past events andemotional intelligence on negative affect, and to determine whether negative affect is related to older people's wellbeing.

Method. The Aging in Spain Longitudinal Study, Pilot Survey (ELES-PS) database wasused. The sample comprised 1747 participants ( $55.9 \%$ women), with ages between 50 to98 years-old $(M=66.2, S D=10.6)$. Stressful past events were evaluated with a list of tenstressful events developed by the project team. Three items of the Trait-Meta-Mood scalewere used to assess emotional intelligence. In order to evaluate negative emotions, the PANAS scale of negative experience was used. Finally, life satisfaction was assessed using the Personal Wellbeing Index.
\end{abstract}


Results. The results of the SEM model indicated that emotional intelligence predicted negative affect, and this in turn was related to participants wellbeing. However past stressful events showed no effect on either the emotional intelligence or on participants' negative affect. Age was used as a control variable and influenced negative affect but didnot impact stressful past events.

Discussion. Internal resources such as emotional intelligence appears to be more important than stressful past events in predicting successful aging.

\title{
Keywords: stressful events, emotional intelligence, negative affect, life satisfaction, older people
}

\section{8 - LATE ONSET BIPOLAR DISORDER: CASE REPORT AND LITERATURE REVIEW}

\author{
Joana Regala ${ }^{1}$, João Reis ${ }^{2}$
}

1- Centro Hospitalar Psiquiátrico de Lisboa (CHPL), Lisbon, Portugal.

2- Department of Geriatric Psychiatry, Centro Hospitalar Psiquiátrico de Lisboa (CHPL), Lisbon, Portugal.

\section{Background:}

Late-onset bipolar disorder (LOBD) remains an incompletely understood nosological entity, in reason of its complexity and the paucity of research in this issue. It is not yet clear whether LOBD is a "phenocopy" of the classic early-onset bipolar disorder (EOBD), sharing symptoms but having a different aetiology, or whether both have a common underlying vulnerability that interacts with age-specific triggering factors. Some authors have proposed that LOBD is a heterogeneous entity, comprising secondary mania (including organic brain disease), bipolarity in the context of dementia-like processes (BD type VI), and LOBD as expression of a lower vulnerability to bipolarity.

\section{Case Report:}

Female patient with previous medical history of hypertension and dyslipidaemia, and psychiatric history of recurrent severe depressive episodes since early age, with melancholic and psychotic features, had a

first hypomanic episode at 76 years-old, under treatment with tricyclic antidepressant and electroconvulsive therapy. Meanwhile, she suffered a traumatic brain injury (TBI) complicated with subdural and subarachnoid hematoma, as well as intraventricular haemorrhage, which is an indirect sign of diffuse axonal injury (DAI). Later, at 79 years-old, she presented a mixed episode characterized by racing thoughts, flight of ideas, non-systematized persecutory and ruin delusions, hyposomnia, and ultradian alternation between dysphoric and depressive mood, psychomotor agitation and retardation, emotional lability, hypersyntonic contact and hostility. Medical conditions that could account for secondary mania were ruled out. This clinical picture subsided within few weeks under treatment with olanzapine and electroconvulsive therapy. After recovery memory deficits were noticed.

\section{Concluding remarks:}

This case illustrates a "latent" BD, in a patient with previous recurrent depressive disorder, manifesting the first episode of mania in late life. Several triggering factors may have contributed to this longitudinal evolution, lowering the threshold to manifest mania, namely cerebrovascular risk factors, a possible underlying degenerative process and DAI secondary to TBI, which through structural dysconnectivity also contributes to cognitive dysfunction. The deleterious effect of recurrent and severe depressive episodes on cognition is well documented. The question of whether some forms of LOBD could constitute a special risk factor for developing dementia deserves further investigation. 